\title{
Logarithmically Complete Monotonicity of a Function Involving the Gamma Functions
}

\author{
Mohammad Soueycatt, Abedalbaset Yonsoo, Ahmad Bekdash, Nabil Khuder Salman*
}

Department of Mathematics, Faculty of Science, Tishreen University, Lattakia, Syria

Email address:

nabildolimi123@gmail.com (N. K. Salman)

${ }^{*}$ Corresponding author

\section{To cite this article:}

Mohammad Soueycatt, Abedalbaset Yonsoo, Ahmad Bekdash, Nabil Khuder Salman. Logarithmically Complete Monotonicity of a Function Involving the Gamma Functions. American Journal of Applied Mathematics. Vol. 8, No. 1, 2020, pp. 17-21.

doi: $10.11648 /$ j.ajam.20200801.13

Received: November 29, 2019; Accepted: December 21, 2019; Published: January 31, 2020

\begin{abstract}
The monotonic functions were first introduced by S. Bernstein as functions which are non-negative with nonnegative derivatives of all orders. He proved that such functions are necessarily analytic and he showed later that if a function is absolutely monotonic on the negative real axis then it can be represented there by a Laplace- Stieitjes integral with nondecreasing determining function and converse. Somewhat earlier F. Hausdorff had proved a similar result for completely monotonic sequences which essentially contained the Bernstein result. Bernstein was evidently unaware of Hausdorff's result, and his proof followed entirely independent lines. Since then many studies have been written on monotonic functions. In this work, we mainly have proved that a certain function involving ratio of the Euler gamma functions and some parameters is completely and logarithmically completely monotonic. Also, we have given the sufficient conditions for this function to be respectively completely and logarithmically completely monotonic. As applications, some inequalities involving the volume of the unite ball in the Euclidian space $\mathrm{R}^{\mathrm{n}}$ are obtained. The established results not only unify and improve certain known inequalities including, but also can generate some new inequalities and the given results could trigger a new research direction in the theory of inequalities and special functions.
\end{abstract}

Keywords: Completely Monotonic, Inequality, Logarithmically Completely Monotonic Function, Gamma Function

\section{Introduction}

A function $f$ is said to be completely monotonic [1] on interval $I \subseteq \mathbb{R}$ if it has derivatives of all orders on $I$ and satisfies for all $u>0$ and $k \in \mathbb{N}$

$$
0 \leq(-1)^{k} f^{(k)}(u)<\infty .
$$

A positive function $f$ is said to be logarithmically completely monotonic (see for example [2]) on an interval $I \subseteq \mathbb{R}$ if it has derivatives of all orders on $I$ and its logarithm $\ln f$ satisfies for $k \in \mathbb{N}$

$$
0 \leq(-1)^{k}[\ln f(u)]^{k}<\infty .
$$

The class of completely monotonic functions on $(0, \infty)$ may be characterized by [1] as:

$f(u)$ is completely monotonic for $0<u<1$ if and only if

$$
f(u)=\int_{0}^{\infty} e^{-s u} d \mu(s),
$$

where $\mu(s)$ is non-decreasing and the integral converges for $0<u<\infty$.

It is proved that $f$ is logarithmically completely monotonic if and only if $f^{\alpha}$ is completely monotonic for all $\alpha>0$ [3].

It is known that any logarithmically completely monotonic function must be completely monotonic, but not conversely [4].

The logarithmically completely monotonic function was characterized as the infinitely divisible completely monotonic functions [5]. Recently, the completely monotonic or logarithmically completely monotonic functions have been the subject of intensive research. For more details we refer the reader to [6]-[12].

The gamma function $\Gamma(u)$ is defined for $u>0$ by the integral 


$$
\Gamma(u)=\int_{0}^{\infty} t^{u-1} e^{-t} d t
$$

The logarithmic derivative of $\Gamma(u)$, denoted by $\psi(u)=$ $\frac{\Gamma^{\prime}(u)}{\Gamma(u)}$, is called the psi or digamma function and the $\psi^{(k)}(u)$ for $k \in \mathbb{N}$ are called the polygamma functions.

The following results were investigated in [13]:

The function

$$
\frac{[\Gamma(u+v+1) / \Gamma(v+1)]^{\frac{1}{u}}}{u+v+1}
$$

is decreasing with respect to $u \geq 1$ for fixed $v \geq 0$.

Consequently, for positive real numbers $u \geq 1$ and $v \geq 0$, we have

$$
\frac{u+v+1}{u+v+2} \leq \frac{[\Gamma(u+v+1) / \Gamma(v+1)]^{\frac{1}{u}}}{[\Gamma(u+v+2) / \Gamma(v+1)]^{\frac{1}{u+1}}}
$$

The function (1) was proved to be logarithmically completely monotonic with respect to $u \in(0, \infty)$ for $v \geq 0$ and so is its reciprocal for $-1<v \leq-\frac{1}{2}$ [14].

Consequently, the inequality (2) is true for $(u, v) \in$ $(0, \infty) \times[0, \infty)$ and reversed for $(u, v) \in(0, \infty) \times\left(-1,-\frac{1}{2}\right]$.

For $(u, v) \in(0, \infty) \times[0, \infty)$ and $\alpha \in[0, \infty)$, the function

$$
\frac{[\Gamma(u+v+1) / \Gamma(v+1)]^{\frac{1}{u}}}{(u+v+1)^{\alpha}}
$$

was proved in [15] to be strictly increasing (or decreasing, respectively) with respect to the single variable $u \in(0, \infty)$ if and only if $0 \leq \alpha \leq \frac{1}{2}$ or ( $\alpha \geq-1$ respectively), to be strictly increasing with respect to $v$ on $[0, \infty)$ if and only if $0 \leq \alpha \leq$ 1 and to be logarithmically concave with respect to the towvariable

$$
(u, v) \in(0, \infty) \times(0, \infty) \text { if } 0 \leq \alpha \leq \frac{1}{4} .
$$

For given $v \in(-1, \infty)$ and $\alpha \in(-\infty, \infty)$, we define the function

$$
h_{\alpha, v}(u)=\left\{\begin{array}{c}
\frac{[\Gamma(u+v+1) / \Gamma(v+1)]^{\frac{1}{u}}}{(u+v+1)^{\alpha}}, u \in(-v-1, \infty) \backslash\{0\} \\
\frac{1}{(v+1)^{\alpha}} e^{\psi(v+1)}, u=0
\end{array}\right.
$$

It is clear that the ranges of $u, v$ and $\alpha$ in the function $h_{\alpha, v}(u)$ extend the corresponding ones in the functions (1) and (3) which were ever discussed in [15].

In this work, we prove that the function $h_{\alpha, v}(u)$ is logarithmically completely monotonic function in some cases.

\section{Results}

Our main results are the following:

Theorem 1. Let $v>-1$. Then

a The function (4) is logarithmically completely monotonic with respect to $(-v-1, \infty)$ if and only if $\alpha \geq \max \left\{1, \frac{1}{v+1}\right\}$.

b If $\alpha \leq \min \left\{1, \frac{1}{2(v+1)}\right\}$, the reciprocal of the function (4) is logarithmically completely monotonic with respect to $u \in(-v-1, \infty)$.

c The necessary condition for the reciprocal of the function (4) to be logarithmically completely monotonic with respect to $u \in(-v-1, \infty)$ is $\alpha \leq 1$.

The theorem 1 extends and generalizes the logarithmically complete monotonicity of the function (1) established in [14] and a part of the results in [15].

From the theorems 1 we get the following corollary:

Corollary 1. For $t>0, v+1>0$ and $u+v+1>0$, the double inequality

$$
\left(\frac{u+v+1}{u+v+t+1}\right)^{\alpha}<\frac{[\Gamma(u+v+1) / \Gamma(v+1)]^{\frac{1}{u}}}{[\Gamma(u+v+t+1) / \Gamma(v+1)]^{\frac{1}{u+t}}}<\left(\frac{u+v+1}{u+v+t+1}\right)^{b}
$$

holds if $\alpha \geq \max \left\{1, \frac{1}{v+1}\right\}$; and $b \leq \min \left\{1, \frac{1}{2(v+1)}\right\}$.

The inequality (5) generalizes and extends the inequality (2) and the main results in $[16,17]$

For $u+v>0$ and $v+1>0$ the inequality

$$
\frac{[\Gamma(u+v+1) / \Gamma(v+1)]^{\frac{1}{u}}}{[\Gamma(u+v+2) / \Gamma(v+1)]^{\frac{1}{u+1}}}<\left(\frac{u+v}{u+v+1}\right)^{\frac{1}{2}}
$$

is true if $u>1$ and reversed if $x<1$ and that the power $\frac{1}{2}$ is the best possible.

Theorem 2. For any $n \in \mathbb{N}$, let $\Omega_{n}=\frac{\pi^{\frac{n}{2}}}{\Gamma\left(1+\frac{n}{2}\right)}$. The following inequalities are true

$$
\begin{gathered}
\sqrt{\frac{n+2}{n+4}}<\frac{\Omega_{n+2}^{1 /(n+2)}}{\Omega_{n}^{1 / n}}<\sqrt[4]{\frac{n+2}{n+4}} \\
\frac{1}{\pi^{2} /(n-2) n} \sqrt{\frac{n+2}{n+4}}<\frac{\Omega_{n+2}^{\frac{1}{n}}}{\Omega_{n}^{1 /(n-2)}}<\frac{1}{\pi^{2 /(n-2) n}} \sqrt[8]{\frac{n+2}{n+4}}, \\
\sqrt{\frac{n+2}{n+3}}<\frac{\Omega_{n+1}^{\frac{1}{n+1}}}{\Omega_{n}^{1 / n}}<\sqrt[4]{\frac{n+2}{n+3}} .
\end{gathered}
$$

In order to prove our main results, the following lemma is needed.

Lemma $1[17,18]$. For $u \in(0, \infty)$ and $k \in \mathbb{N}$, we have

$$
\ln u-\frac{1}{u}<\psi(u)<\ln u-\frac{1}{2 u}
$$

and

$$
\frac{(k-1) !}{u^{k}}+\frac{k !}{2 u^{k+1}}<(-1)^{k+1} \psi^{(k)}(u)<\frac{(k-1) !}{u^{k}}+\frac{k !}{u^{k+1}}
$$

Proof of Theorem 1. For $u \neq 0$, taking the logarithm of $h_{\alpha, v}(u)$ gives

$$
\ln h_{\alpha, v}(u)=\frac{\ln \Gamma(u+v+1)-\ln \Gamma(v+1)}{u}-\alpha \ln (u+v+1) .
$$


A direct differentiation yields

$$
\left[\ln h_{\alpha, v}(u)\right]^{(k)}=\frac{k !}{u^{k+1}} \sum_{i=0}^{k} \frac{(-1)^{k-i} u^{i} \psi^{(i-1)}(u+v+1)}{i !}-\frac{(-1)^{k} k ! \ln (v+1)}{u^{k+1}}-\frac{(-1)^{k-1}(k-1) ! \alpha}{(u+v+1)^{k}}
$$

for $k \in \mathbb{N}$, where $\psi^{(-1)}(u+v+1)$ and $\psi^{(0)}(u+v+1)$ stand for $\ln \Gamma(u+v+1)$ and $\psi(u+v+1)$ respectively. From the relation (8) we get

$$
\left\{u^{k+1}\left[\ln h_{\alpha, v}(u)\right]^{(k)}\right\}^{\prime}=(-1)^{k-1} u^{k}\left[(-1)^{k-1} \psi^{(k)}(u+v+1)-\frac{(k-1) ! \alpha}{(u+v+1)^{k}}-\frac{k !(v+1) \alpha}{(u+v+1)^{k+1}}\right] .
$$

Using the relations (7) and (8) we obtain

$$
\frac{(k-1) !(1-\alpha)}{(u+v+1)^{k}}+\frac{k ![1 / 2-(v+1) \alpha]}{(u+v+1)^{k+1}} \leq \frac{(-1)^{k-1}}{u^{k}}\left\{u^{k+1}\left[\ln h_{\alpha, v}(u)\right]^{(k)}\right\}^{\prime} \leq \frac{(k-1) !(1-\alpha)}{(u+v+1)^{k}}+\frac{k ![1-(v+1) \alpha]}{(u+v+1)^{k+1}}
$$

for $k \in \mathbb{N}, u \neq 0, v \in(-1, \infty)$ and $\alpha \in(-\infty, \infty)$. Therefore,

$$
\frac{(-1)^{k-1}}{u^{k}}\left\{u^{k+1}\left[\ln h_{\alpha, v}(u)\right]^{(k)}\right\}^{\prime}\left\{\begin{array}{l}
\leq 0 \quad \text { if } \alpha \geq 1 \text { and } \alpha \geq \frac{1}{v+1} \\
\geq 0 \quad \text { if } \alpha \leq 1 \text { and } \alpha \leq \frac{1}{2(v+1)}
\end{array}\right.
$$

for $k \in \mathbb{N}, v>1$ and $u \neq 0$.

For $u>0$, the equation (9) means

$$
\left\{u^{2 k}\left[\ln h_{\alpha, v}(u)\right]^{(2 k-1)}\right\}^{\prime}\left\{\begin{array}{l}
\leq 0 \quad \text { if } \alpha \geq 1 \text { and } \alpha \geq \frac{1}{v+1} \\
\geq 0 \quad \text { if } \alpha \leq 1 \text { and } \alpha \leq \frac{1}{2(v+1)}
\end{array}\right.
$$

and

$$
\left\{u^{2 k+1}\left[\ln h_{\alpha, v}(u)\right]^{(2 k)}\right\}^{\prime}\left\{\begin{array}{l}
\leq 0 \quad \text { if } \alpha \geq 1 \text { and } \alpha \geq \frac{1}{v+1} \\
\geq 0 \quad \text { if } \alpha \leq 1 \text { and } \alpha \leq \frac{1}{2(v+1)}
\end{array}\right.
$$

for $k \in \mathbb{N}$. From (8), we get

$$
\lim _{u \rightarrow 0}\left\{u^{k+1}\left[\ln h_{\alpha, v}(u)\right]^{(k)}\right\}^{\prime}=0
$$

For $k \in \mathbb{N}$ and $v>1$. As a result,

$$
\left[\ln h_{\alpha, v}(u)\right]^{(2 k-1)}\left\{\begin{array}{l}
<0 \quad \text { if } \alpha \geq 1 \text { and } \alpha \geq \frac{1}{v+1} \\
>0 \quad \text { if } \alpha \leq 1 \text { and } \alpha \leq \frac{1}{2(v+1)}
\end{array}\right.
$$

and

$$
\left[\ln h_{\alpha, v}(u)\right]^{(2 k)}\left\{\begin{array}{l}
>0 \quad \text { if } \alpha \geq 1 \text { and } \alpha \geq \frac{1}{v+1} \\
<0 \quad \text { if } \alpha \leq 1 \text { and } \alpha \leq \frac{1}{2(v+1)}
\end{array}\right.
$$

for $k \in \mathbb{N}$ and $u \in(0, \infty)$, that is,

$$
(-1)^{k}\left[\ln h_{\alpha, v}(u)\right]^{(k)}\left\{\begin{array}{l}
>0 \quad \text { if } \alpha \geq 1 \text { and } \alpha \geq \frac{1}{v+1} \\
<0 \quad \text { if } \alpha \leq 1 \text { and } \alpha \leq \frac{1}{2(v+1)}
\end{array}\right.
$$

for $k \in \mathbb{N}$ and $u \in(0, \infty)$. Hence, the function (4) is logarithmically completely monotonic with respect to $u$ on $(0, \infty)$ if $\alpha \geq 1$ and $\alpha \geq \frac{1}{v+1}$ and so is the reciprocal of the function (4) if either $0<\alpha \leq 1$ and $\alpha \leq \frac{1}{2(v+1)}$ or $\alpha \leq 0$ and $v>1$.

If $u \in(-v-1,0)$, the equation (9) means

$$
\left\{u^{k+1}\left[\ln h_{\alpha, v}(u)\right]^{(k)}\right\}^{\prime}\left\{\begin{array}{l}
\geq 0 \quad \text { if } \alpha \geq 1 \text { and } \alpha \geq \frac{1}{v+1} \\
\leq 0 \quad \text { if } \alpha \leq 1 \text { and } \alpha \leq \frac{1}{2(v+1)}
\end{array}\right.
$$

for $k \in \mathbb{N}$. By virtue of (10), it follows that

$$
u^{k+1}\left[\ln h_{\alpha, v}(u)\right]^{(k)}\left\{\begin{array}{l}
\leq 0 \quad \text { if } \alpha \geq 1 \text { and } \alpha \geq \frac{1}{v+1} \\
\geq 0 \quad \text { if } \alpha \leq 1 \text { and } \alpha \leq \frac{1}{2(v+1)}
\end{array}\right.
$$

for $k \in \mathbb{N}$, which is equivalent to the fact that the equations (11) and (12) hold for $u \in(-v-1,0)$. As a result, the equation (13) is valid for $k \in \mathbb{N}$ and $u \in(-v-1,0)$. Therefore, the function $h_{\alpha, v}(u)$ has the same logarithmically complete monotonicity properties on $(-v-1,0)$ as on $(0, \infty)$.

Conversely, if $h_{\alpha, v}(u)$ is logarithmically completely monotonic on $(-v-1, \infty)$, then $\left[\ln h_{\alpha, v}(u)\right]^{\prime}<0$ on $(-v-$ $1, \infty)$, which can be simplified as

$$
\begin{aligned}
\alpha & \geq(u+v+1)\left[\frac{1}{u^{2}} \sum_{i=0}^{1} \frac{(-1)^{1-i} u^{i} \psi^{(i-1)}(u+v+1)}{i !}+\frac{\ln \Gamma(v+1)}{u^{2}}\right] \\
& =\frac{1}{u^{2}}\left[(u+v+1) \ln \Gamma(v+1)-(v+1)(u+v+1) \psi(u+v+1)+(u+v+1)^{2} \psi(u+v+1)-(u+v+1) \ln \Gamma(u+v+1)\right] \\
& =\frac{u+v+1}{u}\left[\frac{u \psi(u+v+1)-\ln \Gamma(u+v+1)}{u}+\frac{\ln \Gamma(v+1)}{u}\right]
\end{aligned}
$$


From (6), it is easy to see that

$$
\lim _{u \rightarrow 0^{+}}\left[u^{2} \psi(u)\right]=0
$$

It is known that

$$
\Gamma(u+1)=u \Gamma(u)
$$

For $u>0$. Taking the logarithm on both sides of (18), rearranging and taking limit lead to

$$
\lim _{u \rightarrow 0^{+}}[u \ln \Gamma(u)]=\lim _{u \rightarrow 0^{+}}[u \ln \Gamma(u+1)]-\lim _{u \rightarrow 0^{+}}[u \ln \mathrm{x}]=0
$$

Taking logarithmic derivatives on both sides of (18) yields

$$
\psi(u+1)=\frac{1}{u}+\psi(u)
$$

for $u>0$ and so

$$
\lim _{u \rightarrow 0^{+}}[u \psi(u)]=-1+\lim _{u \rightarrow 0^{+}}[u \psi(u+1)]=-1
$$

Thus, by utilizing (17), (19) and (20), it is revealed that the limit of the function (15) as $u \rightarrow(-v-1)^{+}$, that is, as $u+v+1 \rightarrow 0^{+}$, equals $\frac{1}{v+1}$. By L'Hospital's rule and the double inequality (7) for $k=1$, we have

$$
\lim _{u \rightarrow \infty} \frac{u \psi(u+v+1)-\ln \Gamma(u+v+1)}{u}=\lim _{u \rightarrow \infty}\left[u \psi^{\prime}(u+v+1)\right]=1 .
$$

Hence, the limit of the function (16) as $u \rightarrow \infty$ equals 1 . In a word, a necessary condition for $h_{\alpha, v}(u)$ to be logarithmically completely monotonic is $\alpha \geq 1$ and $\alpha \geq \frac{1}{v+1}$.

If the reciprocal of $h_{\alpha, v}(u)$ is logarithmically completely monotonic, then the inequality (14) is reversed. Since the limit of the function (16) equals 1 as $u \rightarrow \infty$, as showed above, then the necessary condition $\alpha \leq 1$ is obtained.

Proof of Corollary 1. This follows from the monotonicity properties established in Theorem 1.

Proof of Theorem 2. Putting $t=1, v=0$ and $u=\frac{n}{2}$ in (5) we get

$$
\frac{n+2}{n+4}<\frac{\left[\Gamma\left(\frac{n}{2}+1\right)\right]^{\frac{2}{n}}}{\left[\Gamma\left(\frac{(n+2)}{2}+1\right)\right]^{\frac{2}{n+2}}}<\sqrt{\frac{n+2}{n+4}}
$$

and this mean

$$
\sqrt{\frac{n+2}{n+4}}<\frac{\Omega_{n+2}^{1 /(n+2)}}{\Omega_{n}^{1 / n}}<\sqrt[4]{\frac{n+2}{n+4}}
$$

By taking $v=1, t=1$ and $u=\frac{n}{2}-1$ for $n \in \mathbb{N}$ in (5) we obtain

$$
\frac{1}{\pi^{2} /(n-2) n} \sqrt{\frac{n+2}{n+4}}<\frac{\Omega_{n+2}^{\frac{1}{n}}}{\Omega_{n}^{1 /(n-2)}}<\frac{1}{\pi^{2} /(n-2) n} \sqrt[8]{\frac{n+2}{n+4}}
$$

putting $t=\frac{1}{2}, v=0$ and $u=\frac{n}{2}$ in (5) we get

$$
\sqrt{\frac{n+2}{n+3}}<\frac{\Omega_{n+1}^{\frac{1}{n+1}}}{\Omega_{n}^{1 / n}}<\sqrt[4]{\frac{n+2}{n+3}}
$$

\section{Conclusion}

We have established the necessary and sufficient conditions for a certain function involving ratio of the gamma functions to be logarithmically complete monotonicity properties. As a consequence, we derived some inequalities involving the gamma functions. The established results could trigger a new research direction in the theory of inequalities and special functions.

\section{References}

[1] D. V. Widder, The Laplace transform. New York: Princeton University Press, 1941.

[2] A. Grinshpan and M. Ismail, "Completely monotonic functions involving the gamma and $q$-gamma functions," Proc. Am. Math. Soc., vol. 134, no. 4, pp. 1153-1160, 2006.

[3] R. A. Horn, "On infinitely divisible matrices, kernels, and functions," Z. Für Wahrscheinlichkeitstheorie Verwandte Geb., vol. 8, no. 3, pp. 219-230, 1967.

[4] H. Alzer and C. Berg, "Some classes of completely monotonic functions, II," Ramanujan J., vol. 11, no. 2, pp. 225-248, 2006.

[5] C. Berg, "Integral Representation of Some Functions Related to the Gamma Function," Mediterr. J. Math., vol. 1, no. 4, pp. 433-439, 2004.

[6] F. Qi, and B.-N. Guo, "Logarithmically complete monotonicity of Catalan-Qi function related to Catalan numbers," Cogent Math., vol. 3, no. 1, pp. 1-6, 2016.

[7] K. Nantomah and L. Yin, "Logarithmically Complete Monotonicity of Certain Ratios Involving the \$k\$-Gamma Function," 2019.

[8] F. Qi and A.-Q. Liu, "Completely monotonic degrees for a difference between the logarithmic and psi functions," $J$. Comput. Appl. Math., vol. 361, pp. 366-371, 2019.

[9] F. Qi, "Integral representations for multivariate logarithmic polynomials," J. Comput. Appl. Math., vol. 336, pp. 54-62, 2018.

[10] J. El Kamel and K. Mehrez, "A function class of strictly positive definite and logarithmically completely monotonic functions related to the modified Bessel functions," Positivity, vol. 22, no. 5, pp. 1403-1417, 2018.

[11] L. Yin, L.-G. Huang, X.-L. Lin, and Y.-L. Wang, "Monotonicity, concavity, and inequalities related to the generalized digamma function," Adv. Differ. Equ., vol. 2018, no. 1, p. 246, 2018. 
[12] L. Yin, L.-G. Huang, Z.-M. Song, and X. K. Dou, "Some monotonicity properties and inequalities for the generalized digamma and polygamma functions," J. Inequalities Appl., vol. 2018 , no. 249 , pp. 1-13, 2018.

[13] B.-N. Guo and F. Qi, "Inequalities and monotonicity for the ratio of gamma functions," Taiwan. J. Math., vol. 7, no. 2, pp. 239-247, 2003.

[14] F. Qi and B.-N. Guo, "A logarithmically completely monotonic function involving the gamma function," Taiwan. J. Math., vol. 14, no. 4, pp. 1623-1628, 2010.

[15] T.-H. Zhao, Y.-M. Chu, and Y.-P. Jiang, "Monotonic and
Logarithmically Convex Properties of a Function Involving Gamma Functions,” J. Inequalities Appl., vol. 2009, no. 1, p. $728612,2009$.

[16] Y. Yu, "An inequality for ratios of gamma functions," J. Math. Anal. Appl., vol. 352, no. 2, pp. 967-970, Apr. 2009.

[17] B.-N. Guo and F. Qi, "An extension of an inequality for ratios of gamma functions," J. Approx. Theory, vol. 163, no. 9, pp. 1208-1216, 2011.

[18] F. Qi and B.-N. Guo, "Two new proofs of the complete monotonicity of a function involving the psi function," Bull. Korean Math. Soc., vol. 47, no. 1, pp. 103-111, 2010. 